\section{Efficacy and timing of adjunctive therapy in the anti-VEGF treatment regimen for macular oedema in retinal vein occlusion: 12-month real-world result}

PL Lip ${ }^{1}$, P Cikatricis ${ }^{1}$, A Sarmad', EM Damato ${ }^{2}$ R Chavann', A Mitra' ${ }^{1}$, S Elsherbiny ${ }^{3}$, Y Yang ${ }^{4,5}$ and B Mushtaq ${ }^{1}$

\begin{abstract}
Purpose Various combination treatment regimens have been tried to improve the short-term efficacy of intravitreal monotherapy for the treatment of macular oedema (MO) secondary to retinal vein occlusion (RVO). Our study introduces the RandOL protocol (Ranibizumab and Ozurdex with Laser photocoagulation) of initial antiVEGF therapy, controlling recurrent nonischaemic MO with an intravitreal steroid and applying laser therapy to non-perfused retina. We describe our 12-month follow-up experience on timing for adjunctive therapy and real-world effectiveness and safety data.

Methods A retrospective analysis was carried out on 66 consecutive treatment-naive RVO patients with MO who received our RandOL treatment regimen. Baseline visual acuity (VA) and central retinal thickness (CRT) were compared with 12-month result. Results At 12 months, 77\% had significant VA improvement, $52 \%$ had $\geq 3$-line improvement, and $15 \%$ were worse. Significant improvements in CRT were observed in $97 \%$ (baseline median CRT $=531 \mu \mathrm{m}$ (IQR 435-622) reduced to $245 \mu \mathrm{m}$ (IQR 221-351, $P<0.001)$ at 12 months); $76 \%$ achieved a dry fovea at 1 year. Mean number of total injections required was 5.5 (range $2-11$ ) and $6 \%$ required $\geq 9$ injections in 1 year. Although $70 \%$ received additional Ozurdex, $82 \%$ received $\geq 1$ sessions of laser therapy. The BRVO subgroup achieved better VA and CRT improvement at 1 year, but small numbers limit definitive statistical conclusions.
\end{abstract}

Conclusions Our real-world results using a combination treatment protocol for RVOrelated MO achieved similar desirable anatomical and visual outcomes as with a single-agent therapy with less intravitreal re-treatment rates at first year. Randomised controlled studies are needed to evaluate the role of laser and the ideal timing of combination therapy.

Eye (2018) 32, 537-545; doi:10.1038/eye.2017.230; published online 3 November 2017

\section{Introduction}

Retinal vein occlusion (RVO) is the second most common retinal vascular disease after diabetic retinopathy and is estimated to affect 16 million adults worldwide, with a reported prevalence of $4.6 \%$ in those aged $>80$ years. ${ }^{1,2}$ Following the introduction of intravitreal therapies into routine clinical practice, the treatment options for macular oedema (MO) secondary to RVOs have expanded.

The benefits of anti-VEGF therapies (including Bevacizumab, Ranibizumab, and Aflibercept) and intraocular steroid depot (dexamethasone implant) are well established and widely used in the United Kingdom. All agents with the exception of Bevacizumab are licensed and approved by NICE (National Institute for Health and Care Excellence). ${ }^{3-6}$ Although the results from pivotal studies using monotherapy are promising, the need for an average of nine injections in the first year is challenging for service provision as well as patient compliance and clinic attendances. ${ }^{5,7,8}$

As with many published treatment regimens, our departmental audit results on off-label
${ }^{1}$ Birmingham and Midland Eye Centre, City Hospital, Birmingham, UK

${ }^{2}$ Addenbrooke's Hospital, Cambridge, UK

${ }^{3}$ Warwick Hospital, Warwick, UK

${ }^{4}$ Wolverhampton Eye Infirmary, New Cross Hospital, Wolverhampton, UK

${ }^{5}$ School of Health and Life Sciences, Aston University, Birmingham, UK

Correspondence:

PL Lip, Birmingham and Midland Eye Centre, City Hospital, Dudley Road, Birmingham B18 7QH, UK Tel: +44 121 5543801; Fax: +44 1215076791. E-mail: pllipwoo@gmail.com

Received: 4 March 2017 Accepted in revised form: 5 September 2017 Published online:

3 November 2017

Meeting Presentations: Preliminary audit result presented at the Oxford Ophthalmological Congress, Oxford, UK, July 2015 and at EURETINA, Nice, France, September 2015; final audit result presented at the ARVO Annual Meeting, Seattle, WA, USA, May 2016. 
Bevacizumab in treating chronic MO in RVO patients confirmed the practical dilemma and significant variation in deciding on the optimal loading dose and re-treatment regimen. ${ }^{9}$ As the effectiveness of intravitreal monotherapy appears to be short term, discontinuation often sees the rebound of MO. Hence, there is a desire to explore other treatment modalities such as combination therapy, which may address the multifactorial pathophysiological aspects of this disease. ${ }^{10,11}$

Published combination regimens have varied and most offered the addition of compulsory steroids and limited guidance on management pathway. ${ }^{12,13}$ We introduced a clinical treatment pathway for combination therapy for RVO-related MO: the RandOL protocol (Ranibizumab and Ozurdex with Laser photocoagulation) aiming to standardize and optimize our local RVO treatment strategy. This protocol proposes an initial anti-VEGF regimen, followed by an optional intravitreal steroid and/or laser therapy. We hoped to potentially achieve the same desirable anatomical and visual outcomes as with single-agent therapy but with less intravitreal retreatment rates. In this paper, we describe our 12-month follow-up experience on timing for adjunctive therapy and real-world effectiveness and safety data.

\section{Methods}

This paper reports the audited results on all consecutive new RVO patients who attended the RVO clinic implementing the new RandOL protocol treatment regimen between November 2013 to April 2014 at one hospital (Birmingham and Midland Eye Centre). Retrospective data collection included demographics, past medical and ocular histories, and adverse effects. Our primary outcomes were changes in visual acuity (VA) and central retinal thickness (CRT) after the initial loading dose of anti-VEGF and at 1 year, when compared with baseline. Other recorded data included the frequency of injections and interval of adjunctive therapies over a follow-up period of 12 months. Standard clinical care and informed consent were obtained from all patients but given this was a service development plan review using a new local treatment protocol for our department, no ethical approval was required, as confirmed by our local Research Governance authority.

Standard 'Good Clinical Practice' applied to all patients when obtaining consent for investigations and procedures. A preliminary audit was also performed on the first 40 patients to assess the effectiveness and safety of the protocol, and results were presented and discussed in our local clinical governance meeting. Our 12-month real-world results are based on the first 66 consecutive treatment-naive RVO patients with established MO.

\section{Clinical evaluation and intravitreal procedure}

All RVO referrals were first assessed in a dedicated RVO diagnosis clinic and subsequently managed in the RVO review clinic. Clinical evaluation at every clinic visit included Snellen chart visual acuity (VA) measurement, slit-lamp biomicroscopy, Goldmann tonometry for intraocular pressure (IOP) measurement, and central retinal thickness (CRT) as quantitative measurement of MO using spectral-domain optical coherence tomography (3D-OCT 2000, Topcon, Tokyo, Japan). Wide-field fundus fluorescein angiogram (FFA), using wide-field Optos200Tx scanning laser Ophthalmoscope (Optos PLC, Dunfemline, UK), was also performed at baseline and repeated when clinically indicated.

All patients were re-assessed at 4 weeks following any intravitreal injection to assess VA, CRT, and IOP. Subsequently, stable patients (resolved MO) were reviewed at an interval of 6 weeks (if they received an anti-VEGF) or 3 months (if they received a steroid). Intravitreal injections were performed using aseptic technique and topical Chloramphenicol $0.5 \%$ was used after the procedure for 1 week.

\section{RandOL protocol}

The RandOL protocol is a combination therapy regimen of initial anti-VEGF therapy, focussing on eliminating residual retinal ischaemia by means of laser therapy and controlling recurrent non-ischaemic MO using an intravitreal steroid. At the start of our new patient management pathway in November 2013, the NICEapproved intravitreal agents available for the treatment of RVO-related MO were Ranibizumab (Lucentis $0.5 \mathrm{mg}$ in $0.05 \mathrm{ml}$, Novartis, Basel, Switzerland) and dexamethasone implant (Ozurdex $0.7 \mathrm{mg}$, Allergan, Irvine, CA, USA).

Figure 1 is the RandOL protocol flow chart, which depicts an initial loading dose of three intravitreal Ranibizumab injections at 4 weekly intervals. Additional Ranibizumab would be indicated for those with an insufficient response or recurrence of $\mathrm{MO}$ in the evidence of persistent ischaemia. Dexamethasone implant is indicated for recurrent $\mathrm{MO}$ only in the non-ischaemic retina (as established by wide-field FFA) at any timescale. Generally, dexamethasone implant was to be avoided in glaucoma patients with preexisting extensive visual field loss secondary to advanced glaucomatous optic disc cupping and patients with refractory intra-ocular pressure control.

\section{Laser intervention}

The RandOL protocol advised application of angiography-guided laser therapy on areas of non- 
perfused retina only: $360^{\circ}$ pan-retinal photocoagulation (PRP) for CRVO; sector PRP and/or macular grid laser (omitting central fovea zone) for HRVO and BRVO. Laser

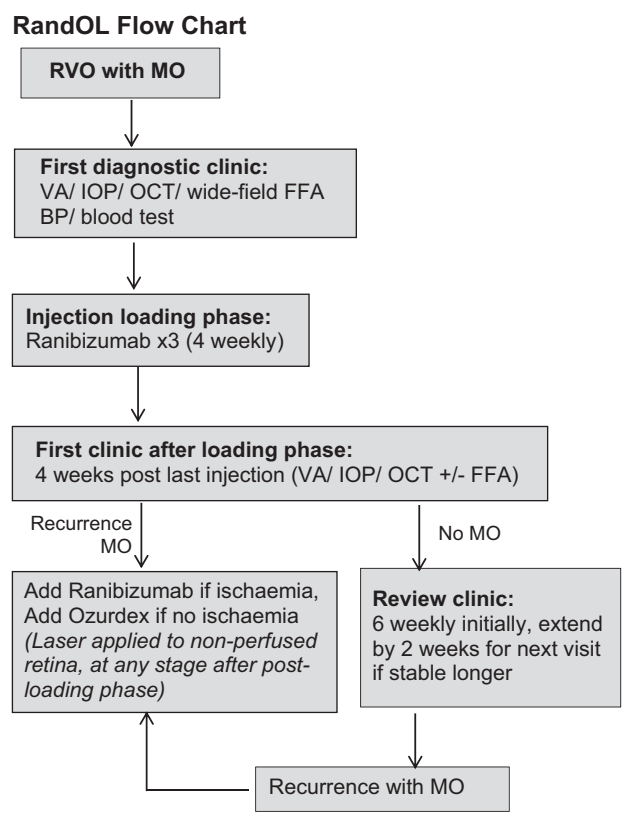

Guidance for intravitreal agent choice in recurrence MO:

Ranibizumab is to be continued in the presence of clinical or angiographic retinal ischaemia (macular or peripheral). The number of re-injections is dependent on the scale of recurrence MO (e.g 1 to 3 injections, 4 weekly) decided by treating clinician.

Dexamethasone implant (Ozurdex) is the choice in the absence of retinal ischaemia (confirmed by FFA, independent of any laser application), and is repeatable after 4month as decided by treating clinician

$V A=V i s u a l$ acuity, $I O P=$ Intraocular pressure check, OCT=Optical coherence tomography, FFA= Fundus fluorescein angiography, $B P=B l o o d$ pressure. Ozurdex= dexamethasone implant

Figure 1 RandOL protocol flow chart for treating retinal vein occlusions (RVO) with macular oedema (MO). treatment was decided at first clinic review after the completion of Ranibizumab loading phase and repeated at different visits if FFA confirmed residual ischaemia. All laser photocoagulation was performed by different laser operators (clinicians) applying laser-machine-specific laser settings, using conventional Argon laser machine or pattern scanning laser (Valon TT Multispot Lasers, Valon Lasers, Finland), which are available in the department during the intervention period.

\section{Data analyses}

Descriptive statistics were used to show demographics and baseline characteristics.

Snellen fractions were converted to non-continous $\log$ MAR (logarithm of the minimum angle of resolution) units by a standard conversion method for statistical purposes. ${ }^{14,15}$ Changes in both VA and CRT at various time points from baseline were tested for statistical significance using Wilcoxon signed-rank test. Statistical analyses were performed using Microsoft Excel 2016 for Windows and a $P$-value of $<0.05$ was considered as statistically significant.

\section{Results}

\section{Demographics and baseline characteristics}

There were 98 treatment-naïve RVO patients attended in the study window period of 6 months, with 12-month follow-up data available for only 90 patients as 8 patients had incomplete data ( 6 patients were transferred back to continue their eye-care elsewhere; 2 patients were deceased prior to first intravitreal treatment). From this

Table 1 Demographic and baseline characteristics

\begin{tabular}{|c|c|c|c|c|}
\hline & Total $R V O, \mathrm{n}=66$ & $C R V O, \mathrm{n}=29$ & $H R V O, \mathrm{n}=9$ & $B R V O, \mathrm{n}=28$ \\
\hline Mean age, years (SD; range) & $72(12,47-94)$ & $77(10,53-92)$ & $70(11,49-79)$ & $67(12,47-94)$ \\
\hline \multicolumn{5}{|l|}{ Gender } \\
\hline Male & $32(48 \%)$ & 15 & 4 & 12 \\
\hline Female & $34(52 \%)$ & 14 & 5 & 16 \\
\hline \multicolumn{5}{|l|}{ Race } \\
\hline Caucasian & $47(71 \%)$ & 23 & 5 & 19 \\
\hline Asian & $12(18 \%)$ & 4 & 0 & 8 \\
\hline Black & $7(11 \%)$ & 2 & 4 & 1 \\
\hline \multicolumn{5}{|c|}{ Presenting ocular features and history } \\
\hline Other eye old RVO & $6(9 \%)$ & 1 & 0 & 5 \\
\hline Same eye recurrence & $6(9 \%)$ & 4 & 0 & 2 \\
\hline Known glaucoma & $14(21 \%)$ & 8 & 3 & 3 \\
\hline Newly diagnosed OHT & $17(26 \%)$ & 7 & 4 & 6 \\
\hline Rubeotic glaucoma & $3(5 \%)$ & 3 & 0 & 0 \\
\hline
\end{tabular}

Abbreviations: BRVO, branch retinal vein occlusion; CRVO, central retinal vein occlusion; $\mathrm{HRVO}$, hemi- retinal vein occlusion; OHT, ocular hypertension; $\mathrm{RVO}$, retinal vein occlusion. 
cohort, only 63 patients presenting with initial MO and 3 patients developed MO at a later stage; the primary endpoint results (VA and CRT analyses) are therefore based on data from the total of 66 patients who received the RandOL regimen.

Table 1 describes baseline demographics of the 66 patients with unilateral eye involvement: 29 had CRVO, 28 of BRVO, and 9 of HRVO. Despite a high nonCaucasian population in our hospital catchment area, we observed a higher RVO presentation from Caucasians. Table 1 shows that $5 \%$ of our cohort RVOs presented with severe ocular ischaemic complications of rubeotic glaucoma, and $18 \%$ had history of previous RVO with $9 \%$ recurrence in the same eye (untreated) of $\geq 6$-month duration. In our series, $21 \%$ have glaucoma and $26 \%$ are newly diagnosed glaucoma or ocular hypertension at RVO presentation.

\section{Treatment exposure}

The majority of the treated patients (85\%) received their first intravitreal injection in $\leq 14$ days from first diagnostic clinic. Patients who received RandOL regimen achieved a mean total of 5.5 of all intravitreal injections in 12 months (Figures $2 \mathrm{a}$ and $\mathrm{b}$ ). Only 4 patients $(6 \%)$ needed $\geq 9$ total injections, and 50 (76\%) needed $\leq 6$ injections (Figure $2 b$ ). The mean number of Ranibizumab injections was 4.3 (range 2-11, one patient missed the third loading dose owing to travelling abroad), and the mean number of dexamethasone implant injections was 1 (range 0-2). Of the 66 patients receiving the RandOL regimen, 46 (70\%) were suitable and received additional dexamethasone implant. The timing of introducing the first dexamethasone implant was on average 2.6 months (SD 0.9 , range $1-5$ months) after the completion of the initial Ranibizumab loading phase.

A high number of patients (54/66,82\%) received one or more sessions of laser therapy at any stage during the 12-month review (Figure 2a table). Subgroup analyses showed that majority received 1-2 laser sessions (Figure 2c) but frequency of laser sessions would depend on variables (laser machine and operator) and wide-field fluorescein angiogram depiction on laser adequacy. The three patients who presented with rubeotic glaucoma with ischaemic retina received $\geq 9$ injections of only Ranibizumab plus extensive laser PRP of 4-5 sessions and required additional cyclodiode laser therapy to control the IOP.

\section{Visual outcomes and subgroup analyses}

Table 2 shows baseline median VA in LogMAR units was 0.71 (Snellen 6/30), which improved to 0.48 (Snellen 6/18) at postloading review, which was maintained at the a Frequency of combination therapy over 12 months

\begin{tabular}{|l|l|l|l|l|}
\hline & $\begin{array}{l}\text { Total RVO } \\
\mathrm{n}=66\end{array}$ & $\begin{array}{l}\text { CRVO } \\
\mathrm{n}=29\end{array}$ & $\begin{array}{l}\text { HRVO } \\
\mathrm{n}=9\end{array}$ & $\begin{array}{l}\text { BRVO } \\
\mathrm{n}=28\end{array}$ \\
\hline $\begin{array}{l}\text { Total injections } \\
\text { Mean frequency (SD) } \\
\text { Mode (range) }\end{array}$ & $\begin{array}{l}5.5(1.8) \\
5(2-11)\end{array}$ & $\begin{array}{l}5.9(2.0) \\
5(2-11)\end{array}$ & $\begin{array}{l}5.2(1.5) \\
7(3-7)\end{array}$ & $\begin{array}{l}5(1.6) \\
4(3-9)\end{array}$ \\
\hline $\begin{array}{l}\text { Ranibizumab only } \\
\text { Mean frequency (SD) } \\
\text { Mode (range) }\end{array}$ & $\begin{array}{l}4.3(1.8) \\
3(2-11)\end{array}$ & $\begin{array}{l}4.7(2.1) \\
3(2-11)\end{array}$ & $\begin{array}{l}4.3(1.3) \\
6(3-6)\end{array}$ & $\begin{array}{l}4.0(1.5) \\
3(3-8)\end{array}$ \\
\hline $\begin{array}{l}\text { Ozurdex only } \\
\text { Mean frequency (SD) } \\
\text { Mode (range) }\end{array}$ & $\begin{array}{l}1.1(0.8) \\
1(0-2)\end{array}$ & $\begin{array}{l}1.1(0.9) \\
2(0-2)\end{array}$ & $\begin{array}{l}0.8(0.7) \\
1(0-2)\end{array}$ & $\begin{array}{l}1.0(0.8) \\
1(0-2)\end{array}$ \\
\hline Laser Procedures & $54(82 \%)$ & $24(83 \%)$ & $7(78 \%)$ & $23(82 \%)$ \\
\hline
\end{tabular}

$R V O=$ Retinal vein occlusion, $C R V O=$ Central retinal vein occlusion, $H R V O=H e m i$ retinal vein occlusion, $B R V O=$ Branch retinal vein occlusion, $S D=S t a n d a r d$ deviation Ozurdex= dexamethasone implant

b Number of total injections received in RVO cohort in 12 months

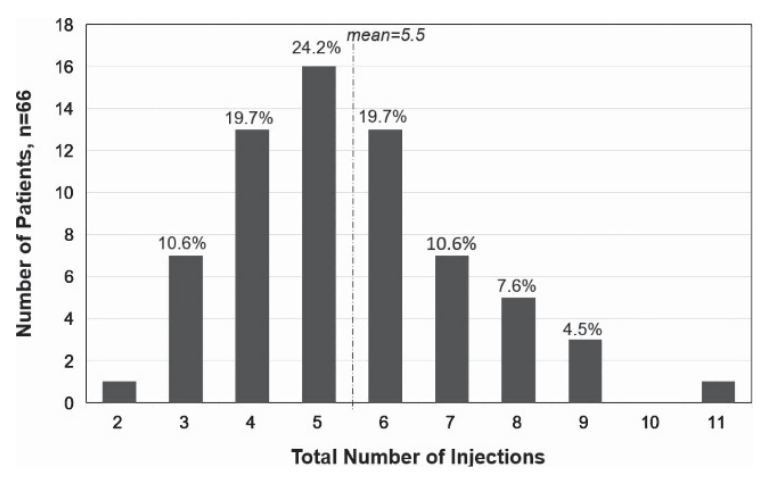

C Number of laser sessions received by RVO subgroups in 12 months

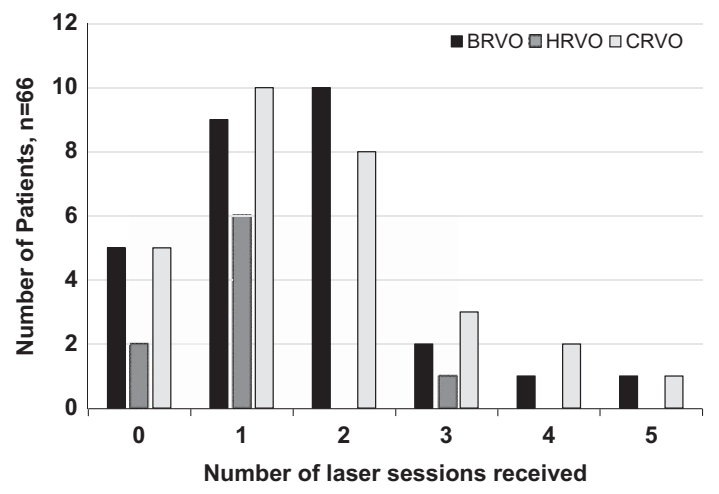

Figure 2 Treatment exposure over 12 months. (a) Frequency of combination therapy over 12 months. (b) Number of total injections received in the RVO cohort in 12 months. (c) Number of laser sessions received by RVO subgroups in 12 months.

month 12 visit $(P<0.001)$. At 1 year, $77 \%$ of patients had vision improvement, $52 \%$ improved by at least three lines. The number of patients achieving 6/12 vision or better was $45.5 \%(30 / 66)$ at 1 year, a significant increase compared with baseline of $19.7 \%$ (13/66 patients). Similar results were obtained when the analysis was performed separately for each RVO subgroup except for the CRVO 
Table 2 Comparison of changes in vision and central retinal thickness: baseline $v s$ year and subgroup analyses

\begin{tabular}{|c|c|c|c|c|}
\hline & Total $R V O, \mathrm{n}=66$ & $C R V O, \mathrm{n}=29$ & $H R V O, \mathrm{n}=9$ & $B R V O, \mathrm{n}=28$ \\
\hline \multicolumn{5}{|c|}{ Comparison of visual acuity (VA) in LogMAR (Snellen equivalent) } \\
\hline Baseline: median VA (IQR) & $0.71(6 / 30)(0.43-1.16)$ & $1.00(6 / 60)(0.52-1.41)$ & $0.72(6 / 30)(0.41-1.05)$ & $0.60(6 / 24)(0.3-0.78)$ \\
\hline aPostloading: median VA (IQR) & $\begin{array}{c}0.48(6 / 18)(0.18-0.84) \\
P<0.001\end{array}$ & & & \\
\hline One year: median VA (IQR) & $\begin{array}{c}0.48(6 / 18)(0.18-0.78) \\
P<0.001\end{array}$ & $\begin{array}{c}0.78(6 / 36)(0.48-1.14) \\
P=0.055\end{array}$ & $\begin{array}{c}0.44(6 / 15)(0.15-0.54) \\
P=0.033\end{array}$ & $\begin{array}{c}0.54(6 / 20)(0.30-0.78) \\
P<0.001\end{array}$ \\
\hline \multicolumn{5}{|l|}{ One year of visual changes } \\
\hline Worse & $10(15 \%)$ & $8(28 \%)$ & $1(11 \%)$ & $1(4 \%)$ \\
\hline Stable & $5(8 \%)$ & $2(7 \%)$ & $1(11 \%)$ & $3(11 \%)$ \\
\hline Improved & $51(77 \%)$ & $19(66 \%)$ & $7(78 \%)$ & $17(61 \%)$ \\
\hline Improved by 3 lines & $34(52 \%)$ & $13(45 \%)$ & $4(44 \%)$ & $16(57 \%)$ \\
\hline Achieved $6 / 12$ or better & $30(45.5 \%)$ & & & \\
\hline \multicolumn{5}{|c|}{ Comparison of central retinal thickness (CRT) changes in $\mu \mathrm{m}$} \\
\hline Baseline: median CRT (IQR) & $531(435-622)$ & $571(466-663)$ & $513(430-559)$ & $499(403-553)$ \\
\hline aPost loading: median CRT (IQR) & $\begin{array}{l}239(215-290) \\
P<0.001\end{array}$ & & & \\
\hline One year: median CRT (IQR) & $\begin{array}{l}245(221-351) \\
P<0.001\end{array}$ & $\begin{array}{l}236(208-332) \\
P<0.001\end{array}$ & $\begin{array}{l}285(243-386) \\
P=0.009\end{array}$ & $\begin{array}{l}252(224-347) \\
P<0.001\end{array}$ \\
\hline
\end{tabular}

Abbreviations: $\mathrm{BRVO}$, branch retinal vein occlusion; IQR, interquartile-range (statistical analysis using Wilcoxon signed-rank test); CRVO, central retinal vein occlusion; HRVO, hemi-retinal vein occlusion; RVO, retinal vein occlusion. ${ }^{a}$ Subgroup analyses on VA and CRT were not performed in the postloading phase.

Table 3 Adverse effects and other ocular procedures in the cohort

\begin{tabular}{lc}
\hline Ozurdex-related complications & \\
Raised intraocular pressure $>21 \mathrm{~mm} \mathrm{Hg}$ & $19 / 46(41 \%)$ \\
Vitreous haemorrhage & $2 / 46(4 \%)$ \\
Cataract related to injection procedure & 0 \\
Cataract related to Ozurdex & Unknown \\
& \\
Cataract operation and other procedures & $12 / 66(18 \%)$ \\
Cataract surgeries & $6 / 12(50 \%)$ \\
RVO retinopathy worsened after cataract sugery & 5 \\
Laser iridotomies/trabeculoplasty & 3 \\
Cyclodiode laser (for rubeotic glaucoma only) &
\end{tabular}

Progression to rubeotic glaucoma

Endophthalmitis

0

0

Abbreviations: Ozurdex, dexamethasone implant; RVO, retinal vein occlusion.

Total number of RVO patients who received RandOL regimen $=66$.

Total number of RandOL patients who received Ozurdex $=46$.

group, where the visual result at 1 year was of borderline statistical significance $(P=0.055)$.

\section{Anatomical outcomes and subgroup analyses}

Table 2 shows the baseline median CRT was $531 \mu \mathrm{m}$. The median reductions in CRT from baseline at the postloading visit and at month 12 were statistically significant $(P<0.001)$ : 64 patients $(97 \%)$ had improved $\mathrm{CMO}$ at 1 year, $76 \%$ had anatomical achievement of dry fovea (no foveal cyst in OCT depiction).
Subgroup analysis shows a lower presenting CRT of $499 \mu \mathrm{m}$ in BRVO compared with CRVO, and HRVO had a closer CRT presentation to BRVO. All three subgroups had median CRT reductions at 1 year, which was statistically significant.

\section{Adverse events and associated procedures}

There were no cases of endophthalmitis in our series. Raised intraocular pressures (IOP $>21 \mathrm{~mm} \mathrm{Hg}$, or higher than baseline IOP) were not detected after Ranibizumab injections but were recorded 4 weeks after dexamethasone implant injection in 19 of the 46 patients (41\%): 12 of these patients were already on antiglaucoma eye drops (known and treated ocular hypertension or glaucoma), 2 of which had only intraocular pressure spike after second dexamethasone implantation but not after the first. All raised intraocular pressures secondary to dexamethasone implant were controllable by topical therapy alone. The other observed adverse effect related to dexamethasone implant was minor vitreous haemorrhage in two patients following injection and resolved with no further complication (Table 3).

There were five poor controlled glaucoma patients (likely to be the cause of RVO) in this cohort and received Ranibizumab injections; they had undergone early procedures (peripheral iridotomies and selective laser trabeculoplasty) as part of their glaucoma treatment plan by their glaucoma specialist. They did not show evidence of worse IOP spike(s) following intravitreal injections. 
Number of patients needing intraocular surgery over the 12 months are summarized as follows: 12 patients underwent cataract surgery (2 combined with goniolysis). None of the 12 cataracts were secondary to injection trauma, but there was no clear recording if these were steroid-related effects. For the first six patients who underwent uneventful phacoemulsification, five had significant worsening of retinal haemorrhages at first postoperative review (Table 3 ). In the subsequent six patients who underwent intraocular surgery in the latter part of the audit period, the surgery was carefully timed to have (or closely follow) a recent Ranibizumab injection, and these latter patients were not noted to have worsening of retinal hemorrhages or recurrence of RVO at the postoperation visit. Seven patients of this postcataract surgery group accounted for $13 \%$ of the cohort, who achieved $>3$ lines VA improvement in 1 year. Cataract surgery could be safely considered during the 'active treatment period' if the procedure was carefully timed.

\section{Discussion}

In this report, we have described our RandOL protocol, a staged combination treatment regimen for RVO with $\mathrm{MO}$, which was designed by a group of retinal specialists through review of the guidelines and evidence-based literature, and was approved and implemented locally as part of a service development plan, to facilitate our 'realworld' specialist eye service. Staged combination therapy using our RandOL protocol achieved the same desirable anatomical and visual outcomes as with a single-agent therapy with less intravitreal re-treatment rates at 1 year.

Specifically, using a mean of 4.3 Ranibizumab injections and one dexamethasone implant over 12 months, we achieved a median visual improvement of $77 \%$ and $52 \%$ had $>15$-letter gain, with $76 \%$ achieving a completely dry fovea at month 12. Although not a direct head-to-head comparison, these outcomes compare very favourably to the 12-month findings from CRUISE study of 77\% below $250 \mu \mathrm{m}$ (8.8 Ranibizumab injections) and BRAVO study of $84 \%$ below $250 \mu \mathrm{m}$ (8.4 Ranibizumab injections). In our series, the appropriate change in practice on optimal timing of cataract surgery also eliminated the risk of worsening RVO in the second part of the re-audit period when the loop was closed, consistent with our approach of introducing an interim audit design.

Intravitreal steroids and anti-VEGF therapies are known to be effective and may have complementary effects on molecular levels in reducing retinal vascular permeability. ${ }^{16-19}$ The combination of Bevacizumab with dexamethasone implant as treatment for RVO patients had been more frequently explored, showing equally favourable results in vision and anatomical improvement, although studied numbers were small and study follow- up was short. ${ }^{12,13}$ The retrospective SHASTA study compared dexamethasone implant monotherapy vs combination of dexamethasone implant with antiVEGF. ${ }^{20}$ This found that visual outcomes were similar in both groups, and as in our series, they showed significant reduction in the required number of injections in the combination group, whereby the number of anti-VEGF injections after dexamethasone implant reduced to 2.7 over a 6-month period with combination therapy. They also reported a high cataract rate of $7.1-19.5 \%$, which is not unusual with any intravitreal steroid agent. Our series found a cataract surgery rate of $18 \%$, which was higher compared with monotherapy of Ranibizumab studies (CRUISE and BRAVO) of 7\% and GENEVA, which reported a rate of 11 eyes in 163 patients. One explanation was that our series had not excluded any media opacity at presentation, which would have been an exclusion criterion in many prospective trials.

Our study is the first report on Ranibizumab, with an optional dexamethasone implant as treatment paradigm in a real-world setting. A recent prospective trial based on mixed anti-VEGF choice with a fixed injection of dexamethasone implant 2 weeks after anti-VEGF injection had similar favourable visual and anatomical outcomes but exposed all patients to steroid-related adverse effect, resulting in the expected high rate of cataract surgery $(62 \%) .{ }^{21}$ The advantage of our protocol regimen is to individualize patient's treatment response, optimizing adjunctive dexamethasone implant only in the 'suitable' (ie, non-ischaemic retina) hence minimizing the known agent-related adverse effects.

\section{Laser therapy for retinal ischaemia}

Our laser use (that is, aim and delivering method) was different from the focal laser used in diabetic maculopathy whereby the latter was aimed at promoting MO resolution by targeting leaking microaneurysms. Indeed, our laser application was purposefully delivered to non-perfusion retina (identifiable by wide-field FFA) aiming to eliminate ischaemia (hence possible retinal neovascularization) rather than direct effect on inducing $\mathrm{MO}$ resolution. As significantly raised intraocular VEGF levels are detectable in RVO eyes, the rationale of initially applying an anti-VEGF agent (short-acting) followed by laser therapy (permanent effect) to theoretically eliminate retinal ischaemia and to reduce the VEGF level load formed the basis of our RandOL protocol. ${ }^{16-19}$

A few randomised controlled trials, such as the CVOS study and more recent case series, have attempted to improve MO through eliminating peripheral retina ischaemia by laser therapy alone or as an adjunctive therapy. ${ }^{11,22,23}$ Singer's case report favours this approach by reporting a single rubeotic glaucoma patient who 
achieved resolution of co-existing rebound $\mathrm{MO}$ following extensive peripheral laser treatment. ${ }^{11}$ In the CVOS study, 155 eyes with CRVO receiving laser monotherapy went on to demonstrate angiographic evidence of reduced MO, but this was not correlated with improved vision and did not support the use of laser for $\mathrm{MO}$ in CRVO. ${ }^{22}$ Nevertheless, Spade's small series of 10 CRVO patients was possibly the closest to sharing our laser strategy (peripheral laser on non-perfused retina guided by widefield angiography). ${ }^{23}$ The author compared changes in $\mathrm{VA}$ and injection frequency in the 6 months preceding the once-off laser treatment vs 6 months after and reported no reduction in Ranibizumab injection frequency nor VA improvement in that small patient cohort with short follow-up duration. ${ }^{23}$ Similar results were found by the RELATE trial, a larger randomised controlled trial investigating different dosages of Ranibizumab arms (allowing rescue laser after month 6) vs Ranibizumab alone. ${ }^{24}$ Arguably, other variables that are much difficult to investigate such as duration of CRVO and the extent of peripheral ischaemia may have influence on the effectiveness and frequency of laser therapy.

Although direct comparisons are not possible with our cohort, there have been a few large randomised trials reporting more encouraging results on combined therapy with initial anti-VEGF injections vs laser alone and allowing a later regime of combined therapy in BRVO patients. ${ }^{25,26}$ For example, the VIBRANT study concluded substantial VA improvement at week 52 when rescue treatment was allowed after week 24 (laser added to Aflibercept-arm, aflibercept to laser-arm), suggesting a potential benefit of the combination regimen. ${ }^{25}$ However, the BRIGHTER study concluded that Ranibizumab with or without laser had superior improvement in VA compared with the laser alone arm at 6 months and further suggested that VA gain was not affected by retinal ischaemic status in BRVO. ${ }^{26}$

The benefits of adjunctive peripheral/macular laser on non-perfused retina of RVO patients are still debatable. Although published randomised clinical trials have concluded that 'laser to non-perfused retina' may have no effect on visual outcome, there are few studies focussed on establishing the role of laser as a preventative measure from retinal neovascularizations. From our own experience in the past few years, there are increasing numbers of late complications (retinal neovascularization and vitreous haemorrhage) presented to eye casualty from untreated RVO or RVO treated with solely antiVEGF therapy. This raises a dilemma for clinicians in deciding if anti-VEGF therapy alone could halt the progression of late RVO complications or would laser remain the ultimate answer for long-term prevention? Randomised controlled trials are therefore needed to evaluate the role of laser on ischaemic RVO.

\section{Poor visual outcome group}

Information on the poor visual outcome group is difficult to obtain when most studies and clinical trials usually exclude patients with advanced severity in their clinical presentation. Although the numbers are small, our subanalysis identified that 13 of the 66 RVO patients (19\%) who had poor visual outcome (worse than 6/60) at 12 months, all shared one or more of these three presenting features: rubeotic glaucoma, established relative afferent pupillary defect, or initial dense haemorrhagic RVO. All except one patient in this poor vision group presented with CRVO. This subgroup was also older (by a mean of 10 years) compared with the age characteristics of our cohort overall, with more medical co-morbidities. The poorer treatment outcome in this severe disease spectrum is also closely related to the poor clinic attendance, as disability from systemic medical problems may prevent patients from receiving the optimal treatment regimen.

In this series, the protocol successfully reversed the visual outcome of one of the three established rubeotic glaucoma patients. Although the range of presenting CRT measurements in this subgroup was very wide (233$961 \mu \mathrm{m})$, almost half $(5 / 13)$ had a CRT of $>600 \mu \mathrm{m}$. However, the CRT at presentation may not, at least in isolation, be as strong a prognostic factor as might intuitively be expected. Nevertheless, the CRT (at 12 months) of this subgroup majority showed fovea thinning of $<200 \mu \mathrm{m}$ as a contributory factor to poor visual outcome.

The main strength of this study is the availability of prospectively planned and uniformly collected follow-up data on a consecutive cohort of patients receiving a new local treatment protocol, thus giving strong representation of the actual 'real-world' experience and results, especially when it also included the spectrum of subgroup analyses and disease severity. We also delivered a pragmatic treatment regimen, easy to understand and execute by clinicians, including training grades, hence reducing the variation in management plan among clinicians. Our study limitations related to its observational design, without a comparator and the use of non-parametric visual acuity scores derived by conversion from Snellen acuities.

In conclusion, our real-world result using a combination treatment protocol for RVO-related MO achieved similar desirable anatomical and visual outcomes as with a single-agent therapy with less intravitreal re-treatment rates at first year. The reduced number of injections with combination regimen may translate to significant benefits for patients, health-care providers, and commissioners. Although the protocol provides a rationale for optional adjunctive therapy based 
on the elimination of ischaemia at different stages of treatment pathway, the benefits of laser to areas of nonperfused retina remains debatable in relation to altering visual outcomes or preventing late complications, such as retinal neovascularization. Randomised controlled studies are needed to evaluate these issues and to address the ideal timing of combination therapy.

\section{Summary}

What was known before

- Combination therapy to anti-VEGF with/without steroid or anti-VEGF with/without laser.

- Small numbers.

- Mostly short-term result.

\section{What this study adds}

- A guidance on a pragmatic combination treatment regimen for RVO CMO (easy to understand and execute by clinicians, including training grades, reducing the variation in management plan among clinicians).

- Provides actual real-world experience, including subgroup analyses/disease severity.

\section{Conflict of interest}

PLL is a speaker for Norvatis. PC is a travel-sponsorship recipient from Allergan and Norvatis. EMD is a speaker for Norvatis. AM is an Advisory Board Member of and speaker for Alimera Sciences. SE is an Advisory Board Member of and speaker for Alimera Science, Bayer, Novartis, and Alcon. YY is an Advisory Board Member of and speaker for Alimera Science, Allergan, Bayer, Novartis, Pfizer, and Thrombogenics. BM is an Advisory Board Member of and speaker for Alimera Science, Allergan, Bayer, Novartis, and ORAYA therapeutics. The other authors declare no conflict of interest.

\section{References}

1 Wong TY, Scott IU. Retinal-vein occlusion. N Eng J Med 2010; 363: 2135-2144.

2 Klein R, Moss SE, Meuer SM, Klein BE. The 15-year cumulative incidence of retinal vein occlusion: the Beaver Dam Eye Study. Arch Ophthalmol 2008; 126(4): 513-518.

3 Epstein DL, Algvere PV, von Wendt G, Seregard S, Kvanta A. Benefit from bevacizumab for macular edema in central retinal vein occlusion: twelve-month results of a prospective, randomized study. Ophthalmology 2012; 119(12): 2587-2591.

4 Heier JS, Campochiaro PA, Yau L, Li Z, Saroj N, Rubio RG et al. Ranibizumab for macular edema due to retinal vein occlusions: long-term follow-up in the HORIZON trial. Ophthalmology 2012; 119(4): 802-809.

5 Heier JS, Clark WL, Boyer DS, Brown DM, Vitti R, Berliner AJ et al. Intravitreal aflibercept injection for macular edema due to central retinal vein occlusion: two-year results from the COPERNICUS study. Ophthalmology 2014; 121(7): 1414-1420.

6 Haller JA, Bandello F, Belfort Jr R, Blumenkranz MS, Gillies M, Heier J et al. Dexamethasone intravitreal implant in patients with macular edema related to branch or central retinal vein occlusion twelve-month study results. Ophthalmology 2011; 118(12): 2453-2460.

7 Brown DM, Campochiaro PA, Singh RP, Li Z, Gray S, Saroj $\mathrm{N}$ et al. Ranibizumab for macular edema following central retinal vein occlusion: six-month primary end point results of a phase III study. Ophthalmology 2010; 117(6): 1124-1133.

8 Brown DM, Campochiaro PA, Bhisitkul RB, Ho AC, Gray S, Saroj $\mathrm{N}$ et al. Sustained benefits from ranibizumab for macular edema following branch retinal vein occlusion: 12-month outcomes of a phase III study. Ophthalmology 2011; 118(8): 1594-1602.

9 Lip PL, Malick H, Damer K, Elsherbiny S, Darrad KM, Mushtaq B et al. One year outcome of bevacizumab therapy for chronic macular oedema in branch and central retinal vein occlusions in the real world clinical practice in the United Kingdom. Clin Ophthalmol 2015; 9: 1779-1784.

10 Matsumoto Y, Freund KB, Peiretti E, Cooney MJ, Ferrara DC, Yannuzzi LA. Rebound macular edema following bevacizumab (Avastin) therapy for retinal venous occlusive disease. Retina 2007; 27(4): 426-431.

11 Singer MA, Tan CS, Suprapaneni K, Sadda S. Targeted photocoagulation of peripheral ischaemia to treat rebound edema. Clin Ophthal 2015; 9: 337-341.

12 Singer MA, Bell DJ, Woods P, Pollard J, Boord T, Herro A et al. Effect of combination therapy with bevacizumab and dexamethasone intravitreal implant in patients with retinal vein occlusion. Retina 2012; 32(7): 1289-1294.

13 Maturi RK, Chen V, Raghinaru D, Bleau L, Stewart MW. A 6-month, subject-masked, randomized controlled study to assess efficacy of dexamethasone as an adjunct to bevacizumab compared with bevacizumab alone in the treatment of patients with macular edema due to central or branch retinal vein occlusion. Clin Ophthalmol 2014; 8: 1057-1064.

14 Gregori NZ, Feuer W, Rosenfeld PJ. Novel method for analyzing Snellen visual acuity measurements. Retina 2010; 30(7): 1046-1050.

15 Snellen-logMAR Visual Acuity Calculator. Available from http:/ / www.myvisiontest.com/logmar.php.

16 Nehme A, Edelman J. Dexamethasone inhibits high glucose-, TNF-alpha-, and IL-1beta-induced secretion of inflammatory and angiogenic mediators from retinal microvascular pericytes. Invest Ophthalmol Vis Sci 2008; 49(5): 2030-2038.

17 Boyd SR, Zachary I, Chakravarthy U, Allen GJ, Wisdom B, Cree IA et al. Correlation of increased vascular endothelial growth factor with neovascularisation and permeability in ischaemic central retinal vein occlusion. Arch Ophthamol 2002; 120: 1644-1650.

18 Koss MJ, Pfister M, Rothweiler F, Michaelis M, Cinatl J, Schubert $\mathrm{R}$ et al. Comparison of cytokine levels from undiluted vitreous of untreated patients with retinal vein occlusion. Acta Ophthal 2012; 90: e98-e103.

19 Noma H, Funatsu H, Yamasaki M, Tsukamoto H, Mimura T, Sone $\mathrm{T}$ et al. Pathogenesis of macular edema with branch retinal vein occlusion and intraocular levels of vascular endothelial growth factor and interleukin-6. Am J Ophthalmol 2005; 140: 256-261. 
20 Dugel P, Capone A, Singer M, Dreyer R, Dodwell D, Roth D et al. Two or more dexamethasone intravitreal implants in treatment-naïve patients with macular edema due to retinal vein occlusion: subgroup analysis of a retrospective chart review study. BMC Ophthalmol 2015; 15: 118-125.

21 Singer MA, Jansen ME, Tyler L, Woods P, Ansari F, Jain U et al. Long-term results of combination therapy using antiVEGF agents and dexamethasone intravitreal implant for retinal vein occlusion: an investigational case series. Clin Ophthalmol 2016; 11: 31-38.

22 Evaluation of grid pattern photocoagulation for macular edema in central vein occlusion. The Central Vein Occlusion Study Group M report. Ophthalmology 1995; 102(10): 1425-1433.

23 Spaide RF. Prospective study of peripheral panretinal photocoagulation of areas of nonperfusion in central retinal vein occlusion. Retina 2013; 33(1): 56-62.

24 Campochiaro PA, Hafiz G, Mir TA, Scott AW, Solomon S, Zimmer-Galler I et al. Scatter photocoagulation does not reduce macular oedema or treatment burden in patients with retinal vein occlusion: the RELATE trial. Ophthalmology 2015; 122(7): 1426-1437.

25 Clark WL, Boyer DS, Heier JS, Brown DM, Haller JA, Vitti R et al. Intravitreal aflibercept for macular edema following branch retinal vein occlusion: 52-week results of the VIBRANT study. Ophthalmology 2016; 123(2): 330-336.

26 Tadayoni R, Waldstein SM, Boscia F, Gerding H, Pearce I, Priglinger $\mathrm{S}$ et al. Individualized stabilization criteria-driven ranibizumab versus laser in branch retinal vein occlusion: six-month results of BRIGHTER. Ophthalmology 2016; 123(6): 1332-1334

(c) (i) (-) $\Theta$ This work is licensed under a Creative Commons Attribution-NonCommercialNoDerivs 4.0 International License. The images or other third party material in this article are included in the article's Creative Commons license, unless indicated otherwise in the credit line; if the material is not included under the Creative Commons license, users will need to obtain permission from the license holder to reproduce the material. To view a copy of this license, visit http://creativecommons.org/licenses/by-nc-nd/4.0/

(C) The Author(s) 2018 